\title{
Reduced Models of Albert Algebras
}

\author{
Holger P. Petersson \\ Fachbereich Mathematik, FernUniversität, Lützowstraße 125, D-58084 Hagen, \\ Deutschland \\ e-mail: Holger.Petersson@FernUni-Hagen.de \\ Michel L. Racine \\ Department of Mathematics, University of Ottawa, 585 King Edward, K1N 6N5 \\ Ottawa, Ontario, Canada \\ e-mail: me@albert.mathstat.uottawa.ca (My account)
}

\section{Introduction}

Let $\mathcal{J}$ be an Albert (= central simple exceptional Jordan) algebra over a field $k$. By results due to Serre [25], Rost [22] and the authors [18], there exists a reduced Albert algebra over $k$, uniquely determined up to isotopy, that becomes isotopic to $\mathcal{J}$ whenever scalars are extended to an arbitrary reducing field of $\mathcal{J}$. In the present paper, we go one step further by investigating reduced models of $\mathcal{J}$, i. e., reduced Albert algebras over $k$ that become isomorphic to $\mathcal{J}$ whenever scalars are extended to an arbitrary reducing field of $\mathcal{J}$. Our principal aim is to establish the following result.

Main Theorem. Every Albert algebra has a reduced model which is unique up to isomorphism.

The notion of a reduced model makes sense not just for Albert algebras but for arbitrary absolutely simple Jordan algebras of degree 3 as well (but is nontrivial only if the Jordan algebra in question happens to be a division algebra, forcing it to be an Albert algebra or of dimension 9). In fact, the main theorem continues to hold under these more general circumstances (2.8). As to the proof, uniqueness immediately follows from Springer's Extension Theorem for quadratic forms (1.2). Existence, on the other hand, will be obtained in two steps. In the first step, we establish an intimate relationship between the (still hypothetical) reduced model of an Albert algebra and the one of any of its nine-dimensional absolutely simple subalgebras (2.5) whereas, in the second step, we prove the existence of a reduced model for any nine-dimensional absolutely simple Jordan algebra $A$ of degree 3 
(2.7) by drawing a connection to the octonion algebra, Oct $A$, of $A$ as defined in $[18,1.7]$. The paper then proceeds to derive explicit descriptions of Oct $A$ in terms of the parameters used to build up $A$ by means of the toral Tits process [14], which had been done previously for the norm of Oct $A[18,4.2]$. The approach adopted here uses the Scharlau transfer of quadratic forms and yields the algebra structure of Oct $A$ via the Cayley-Dickson doubling process (3.7). Alternately, we describe Oct $A$ by means of an appropriate ternary hermitian form (3.9). In summary, we thus obtain not only a proof of the main theorem but at the same time an explicit description of the reduced model of $\mathcal{J}$ once $\mathcal{J}$ has been realized by an iterated application of the Tits process $[16,3.1]$.

There are various applications. For one thing, reduced models provide a convenient way of defining the invariants mod 2 introduced by Serre [25] and Rost [22] using different methods (2.10). We also obtain several characterizations of those absolutely simple Jordan algebras of degree 3 and dimension 9 whose octonion algebras are split (2.11). Furthermore, the explicit description we have derived for the reduced model leads to a (comparatively) straightforward construction of Albert division algebras with nonzero invariants mod 2 (4.11), forcing them to be pure second Tits constructions in the sense of [17].

Most results of this paper have been announced in [20]. The authors are indebted to M.-A. Knus and E. Neher for useful conversations on the subject.

\section{Prerequisites}

Let $k$ be an arbitrary base field, remaining fixed throughout. All vector spaces, algebras etc. over $k$ are supposed to be finite-dimensional. For the convenience of the reader we collect here a few results scattered in the literature that are important for understanding the subsequent development.

1.1. Let $q$ be a quadratic form on a vector space $V$ over $k$. $q$ is said to be nonsingular if its induced symmetric bilinear form is nondegenerate in the usual sense. We denote by $\mathbf{h}, \perp,\langle a\rangle$ the hyperbolic plane, the orthogonal sum of quadratic forms, the onedimensional quadratic form determined by $a \in k$, respectively. Writing $k^{\bullet}$ for the mulitplicative group $k^{\times}=k-\{0\}$ if char $k \neq 2$, for the additive group $k$ otherwise, we define $\wp: k^{\bullet} \longrightarrow k^{\bullet}$ by $\wp(\alpha)=\alpha^{2}$ in the first case, $\wp(\alpha)=\alpha+\alpha^{2}$ in the second $\left(\alpha \in k^{\bullet}\right)$. In all characteristics, we view $\Gamma(k)=$ coker $\wp=H^{1}(k, \mathbf{Z} / 2 \mathbf{Z})$ as an additive group. Suppose $q$ is nonsingular and $e \in V$ is a base point of $q$, so $q(e)=1$. Given $\delta \in \Gamma(k)$ and choosing $d \in k^{\bullet}$ representing $\delta$, the quadratic form $q_{\delta}: V \longrightarrow k$ given by 
$q_{\delta}(v)=d q(v)+\frac{1-d}{4} q(e, v)^{2}(\operatorname{char} k \neq 2), q_{\delta}(v)=q(v)+d q(e, v)^{2}(\operatorname{char} k=2)$ up to isometry only depends on $\delta$ and not on the choice of $d, e[18,2.6]$. We call $q_{\delta}$ the $\delta$-associate of $q$ and have

$$
\begin{aligned}
\left(q_{\delta}\right)_{\varepsilon} & \cong q_{\delta+\epsilon}, \\
\left(q \perp q^{\prime}\right)_{\delta} & \cong q_{\delta} \perp \delta^{\prime} q^{\prime}
\end{aligned}
$$

for $\delta, \varepsilon \in \Gamma(k)$ and any nonsingular quadratic form $q^{\prime}$ over $k$, where $\delta^{\prime}=\delta$ (char $k \neq$ $2), \delta^{\prime}=1$ (char $\left.k=2\right)$ [18, 2.7]. For a more general set-up for associates of quadratic forms, see a forthcoming paper by Loos [8]. We will also need the following well known result.

1.2. Springer's Extension Theorem. Let $k^{\prime} / k$ be a field extension of odd degree. If $q, q_{1}$ are nonsingular quadratic forms over $k$ and $q_{2}^{\prime}$ is a nonsingular quadratic form over $k^{\prime}$ such that

$$
q \otimes_{k} k^{\prime} \cong\left(q_{1} \otimes_{k} k^{\prime}\right) \perp q_{2}^{\prime},
$$

then there exists a nonsingular quadratic form $q_{2}$ over $k$, uniquely determined up to isometry, such that

$$
q_{2}^{\prime} \cong q_{2} \otimes_{k} k^{\prime}, q \cong q_{1} \perp q_{2} .
$$

In particular, $q \otimes_{k} k^{\prime} \cong q_{1} \otimes_{k} k^{\prime}$ implies $q \cong q_{1}$.

1.3. Let $C$ be a composition algebra over $k$. Its norm will be denoted by $n_{C}$, its trace by $t_{C}$ and its canonical involution by ${ }^{-}$unless stated otherwise. We will always tacitly assume that $n_{C}$ is nonsingular (1.1).

1.4. A separable commutative associative $k$-algebra (of dimension $n$ ) is called a torus (of rank $n$ ). A torus is said to be split if it is isomorphic to a direct sum of copies of the base field. The discriminant of a torus $E / k$, uniquely determined up to squares, will be noted by $d_{E / k}$. Tori of rank 2 are classified by $\Gamma(k)[18$, 2.8]. We write $K=k\{\delta\}$ for the torus of rank 2 corresponding to $\delta \in \Gamma(k)$ and call $\delta=\delta(K / k)$ the pseudodiscriminant of $K$ since it is the usual discriminant for char $k \neq 2$. On the other hand, $d_{K / k}=1$ for char $k=2$. Finally, by $[18,2.9]$, we have

$$
\left(n_{k\{\delta\}}\right)_{\varepsilon} \cong n_{k\{\delta+\varepsilon\}}
$$

for $\delta, \varepsilon \in \Gamma(k)$. 
1.5. Let $(N, \#, 1)$ be a cubic form with adjoint and base point over $k$, so for some $k$-vector-space $V, N: V \longrightarrow k$ is cubic, $\#: V \longrightarrow V$ is quadratic and $1 \in V$ is a point such that, writing $T=-\left(D^{2} \log N\right)(1)$, the relations $x^{\# \#}=$ $N(x) x, N(1)=1, T\left(x^{\#}, y\right)=(D N)(x) y, 1^{\#}=1,1 \times y=T(y) 1-y(\times$ the bilinearization of $\#, T(y)=T(1, y))$ hold under all scalar extensions. Then the $U$-operator $U_{x} y=T(x, y) x-x^{\#} \times y$ and the base point 1 give $V$ the structure of a unital quadratic Jordan algebra, written as $\mathcal{J}=\mathcal{J}(N, \#, 1)$. Conversely, given any Jordan algebra $\mathcal{J}$ of degree 3 over $k$, with generic norm $N=N_{\mathcal{J}}$ and identity element $1=1_{\mathcal{J}}$, we define the adjoint \# to be the numerator of the inversion map and obtain a cubic form $(N, \#, 1)$ with adjoint and base point satisfying $\mathcal{J}=\mathcal{J}(N, \#, 1)$. We write $T=T_{\mathcal{J}}$ for the associated trace form, which agrees with the generic trace of $\mathcal{J}, S=S_{\mathcal{J}}$ for the quadratic form $x \longmapsto T\left(x^{\#}\right)$, which is called the quadratic trace of $\mathcal{J}$, and have

$$
S(x, y)=T(x) T(y)-T(x, y) \quad(x, y \in \mathcal{J})
$$

by $[9,(16)]$. We also denote by $\mathcal{J}^{0}=\operatorname{ker} T$ the space of trace zero elements in $\mathcal{J}$ and by $S_{\mathcal{J}}^{0}$ the restriction of $S_{\mathcal{J}}$ to $\mathcal{J}^{0}$. Finally, $W^{\perp}$ stands for the orthogonal complement relative to $T_{\mathcal{J}}$ of any subspace $W \subset J$.

1.6. A torus $E / k$ of rank 3 may be viewed canonically as a Jordan algebra of degree 3. We write $\delta(E / k) \in \Gamma(k)$ for the pseudodiscriminant of $E$ and recall that $\delta(E / k)=0$ if and only if $E / k$ splits or is a cyclic field extension [18, 2.8]. As in 1.4 we have $d_{E / k}=1$ for char $k=2$, and $[18,3.3,3.2]$ yield

$$
\begin{aligned}
& S_{E} \cong<-d_{E / k}>\perp \mathbf{h}, \\
& S_{E}^{0} \cong n_{k\{\delta(E / k)+1\}}
\end{aligned}
$$$$
(\operatorname{char} k=2) \text {. }
$$

1.7. Let $\mathcal{J}$ be a Jordan algebra of degree 3 over $k$ which is absolutely simple in the sense that it stays simple under all base field extensions. Then $\mathcal{J}$ is either a division algebra or it is reduced [21, Theorem 1]; in the latter case, $\mathcal{J}$ can be coordinatized, so there exist a composition algebra $C$ over $k$ and a diagonal matrix $g=\operatorname{diag}\left(g_{1}, g_{2}, g_{3}\right) \in \mathrm{GL}_{3}(k)$ such that $\mathcal{J} \cong \mathrm{H}_{3}(C, g)$, the Jordan algebra of 3-by3 matrices $x$ having diagonal entries in $k$, off-diagonal entries in $C$ and satisfying $x=g^{-1 t} \bar{x} g$. The diagonal of $\mathcal{J}=\mathrm{H}_{3}(C, g)$ is a torus $E / k$ of rank 3 , and [18, (24)] yields

$$
\left.S_{\mathcal{J}}\right|_{E^{\perp}} \cong-\left[\left(g_{2} g_{3} n_{C}\right) \perp\left(g_{3} g_{1} n_{C}\right) \perp\left(g_{1} g_{2} n_{C}\right)\right] .
$$

As already observed in [10, p. 594], Springer's isomorphism criterion for reduced Albert algebras [21, Theorem 3] extends to absolutely simple reduced Jordan algebras of degree 3 and shows that two such algebras are isomorphic if and only if 
they have isomorphic coordinate algebras and isometric quadratic traces. Also, $\mathcal{J}$ contains nilpotent elements other than zero if and only if it can be coordinatized as above, with $g=\operatorname{diag}(1,-1,1)$.

1.8. We now describe a special case of the Tits process [15] which yields the most important examples of cubic forms with adjoint and base point. Let $K / k$ be a torus of rank 2, $B$ a separable associative $K$-algebra of degree 3 (with the obvious meaning if $K \cong k \oplus k$ splits) and $*$ a $K / k$-involution of $B$. Write $N$ for the generic norm, $T$ for the generic trace, \# for the adjoint of $B$ and put

$$
A=\mathrm{H}(B, *)=\left\{a \in B: a=a^{*}\right\} .
$$

Suppose further that we are given invertible elements $u \in A, \beta \in K$ satisfying $N(u)=\beta \beta^{*}$. Extending $N, \#, 1=1_{B}=1_{A}$ as given on $B$ and $A$ to the $k$-vector space $V=A \oplus B$ according to the rules

$$
\begin{aligned}
N((a, b)) & =N(a)+\beta N(b)+\beta^{*} N(b)^{*}-T\left(a, b u b^{*}\right), \\
(a, b)^{\#} & =\left(a^{\#}-b u b^{*}, \beta^{*} b^{* \#} u^{-1}-a b\right), \\
1 & =\left(1_{A}, 0\right)
\end{aligned}
$$

for $a \in A, b \in B$, we obtain a cubic form $(N, \#, 1)$ with adjoint and base point over $k$. The ensuing Jordan algebra will be written as $\mathcal{J}=\mathcal{J}(K, B, *, u, \beta)$. It has the associated trace form given by

$$
T(x, y)=T(a, c)+T\left(b u, d^{*}\right)+T\left(d u, b^{*}\right)
$$

for $x=(a, b), y=(c, d) \in \mathcal{J}$, forcing $T(x)=T(a)$ and then

$$
S(x)=T\left(a^{\#}-b u b^{*}\right)
$$

for the quadratic trace of $\mathcal{J}$. Recall that $\mathcal{J}$ is a division algebra if and only if $\beta$ is not a generic norm of $B[15,5.2,5.3]$.

In particular, if $(B, *)$ is a central simple associative $k$-algebra of degree 3 with involution of the second kind (central simplicity always being unterstood in the context of algebras with involution), the preceding construction applies to $K=$ Cent $(B)$, the center of $B$, and $\mathcal{J}(B, *, u, \beta)=\mathcal{J}(K, B, *, u, \beta)$ is an Albert algebra.

1.9. Let $A$ be a separable associative $k$-algebra of degree 3 and $\alpha \in k^{\times}$. Then we may specialize 1.8 to $B=A \oplus A^{\text {op }}$, * the exchange involution, $K=k \oplus k, u=$ $\left(1_{A}, 1_{A}\right), \beta=\left(\alpha, \alpha^{-1}\right)$. By $[15,3.5]$ the ensuing Jordan algebra $\mathcal{J}(K, B, *, u, \beta)$ 
identifies with the first Tits construction $\mathcal{J}(A, \alpha)$ which is defined as follows: We have $\mathcal{J}(A, \alpha)=\mathcal{J}(N, \#, 1)$ where $(N, \#, 1)$ is the cubic form with adjoint and base point on $V=A \oplus A \oplus A$ given by

$$
\begin{aligned}
N(x) & =N_{A}\left(a_{0}\right)+\alpha N_{A}\left(a_{1}\right)+\alpha^{-1} N_{A}\left(a_{2}\right)-T_{A}\left(a_{0} a_{1} a_{2}\right), \\
x^{\#} & =\left(a_{0}^{\#}-a_{1} a_{2}, \alpha^{-1} a_{2}^{\#}-a_{0} a_{1}, \alpha a_{1}^{\#}-a_{2} a_{0}\right), \\
1 & =\left(1_{A}, 0,0\right)
\end{aligned}
$$

for $x=\left(a_{0}, a_{1}, a_{2}\right)$. We also have relation

$$
S_{\mathcal{J}(A, \alpha)}(x)=S_{A}(x)-T_{A}\left(a_{1}, a_{2}\right)
$$

by $[15,(3.2),(3.3)]$.

1.10. Let $L / k, E / k$ be tori of rank 2,3 , respectively. In 1.8 we put $K=L, B=$ $E \otimes_{k} L, *=\mathbf{1}_{E} \otimes^{-}$(cf. 1.3). Then $A=\mathrm{H}(B, *)$ canonically identifies with $E$ and, given $v \in E, \gamma \in L$ invertible such that $N_{E}(v)=\gamma \bar{\gamma}$, we may form the Jordan algebra

$$
\mathcal{J}=\mathcal{J}(E, v, \gamma, L)=\mathcal{J}\left(L, E \otimes_{k} L, \mathbf{1}_{E} \otimes^{-}, v, \gamma\right)
$$

called a toral Tits process [14], which is absolutely simple of degree 3 and dimension 9. Conversely, every absolutely simple Jordan algebra of degree 3 and dimension 9 arises in this way $[16,3.1]$. If $L$ splits, some invertible element $\alpha \in k^{\times}$allows the identification of $\mathcal{J}$ with the first Tits construction $\mathcal{J}(E, \alpha)(1.9)$. In the general situation, we have $A \cong \mathrm{H}\left(B^{\prime}, *^{\prime}\right)$ for some central simple associative $k$-algebra $\left(B^{\prime}, *^{\prime}\right)$ of degree 3 with involution of the second kind, and, setting $K=\operatorname{Cent}\left(B^{\prime}\right)$, [14, Theorem 1] yields

$$
\delta(K / k)=\delta(E / k)+\delta(L / k)
$$

in $\Gamma(k)$, forcing

$$
d_{K / k} \equiv d_{E / k} d_{L / k} \bmod k^{\times 2}
$$

since both sides are trivial in characteristic 2 .

1.11. Let $A$ be an absolutely simple Jordan algebra of degree 3 and dimension 9 over $k$. Then there exists a unique central simple associative $k$-algebra $(B, *)$ of degree 3 with involution of the second kind satisfying $A \cong H(B, *)$. As in $[18,1.7]$, 
we define the octonion algebra of $A$, written as Oct $A$, to be the coordinate algebra of the reduced Albert algebra $\mathcal{J}(B, *, 1,1)$. Setting $K=\operatorname{Cent}(B)$ and choosing any maximal torus $E \subset A,[18,1.9,4.2,4.3]$ yield

$$
\begin{aligned}
n_{\text {Oct } A} & \cong n_{k\{\delta(E / k)+\delta(K / k)\}} \perp\left(\left.d_{K / k} S_{A}\right|_{E^{\perp}}\right), & & \\
d_{K / k} S_{A} & \cong<-1>\perp n_{\text {Oct } A} & & (\operatorname{char} k \neq 2), \\
S_{A}^{0} & \cong\left(n_{\text {Oct } A}\right)_{\delta(K / k)+1} & & (\operatorname{char} k=2) .
\end{aligned}
$$

\section{Reduced models}

2.1. Let $\mathcal{J}$ be an absolutely simple Jordan algebra of degree 3 over $k$. By a reducing field of $\mathcal{J}$ we mean a field extension $k^{\prime} / k$ such that $\mathcal{J} \otimes_{k} k^{\prime}$ is reduced. For example, any cubic subfield of $\mathcal{J}$ has this property. An absolutely simple Jordan algebra $\mathcal{J}_{\text {red }}$ of degree 3 over $k$ is said to be a reduced model of $\mathcal{J}$ if it is reduced and satisfies the relation $\mathcal{J} \otimes_{k} k^{\prime} \cong \mathcal{J}_{\text {red }} \otimes_{k} k^{\prime}$ for any reducing field $k^{\prime} / k$ of $\mathcal{J}$.

Our aim in this paper is to establish existence and uniqueness of reduced models and to provide an explicit description in terms of the Tits process. Uniqueness immediately follows from Springer's isomorphism criterion for Jordan algebras of degree 3 (1.7) combined with his extension theorem for quadratic forms (1.2). To establish existence, we start with the following lemma.

2.2. Lemma. Let $\mathcal{J}$ be an absolutely simple Jordan algebra of degree 3 over $k$. Then there exists a nonsingular quadratic form $Q_{\mathcal{J}}$ over $k$, uniquely determined up to isometry, such that

$$
\begin{aligned}
& S_{\mathcal{J}} \cong<-1>\perp \mathbf{h} \perp Q_{\mathcal{J}} \\
& \text { (char } k \neq 2) \text {, } \\
& S_{\mathcal{J}}^{0} \cong n_{k\{1\}} \perp Q_{\mathcal{J}} \\
& \text { (char } k=2) \text {. }
\end{aligned}
$$

Furthermore, if $\mathcal{J}$ is reduced, given in the form $\mathcal{J}=\mathrm{H}_{3}(C, g)$ for some composition algebra $C$ over $k$ and some $g=\operatorname{diag}\left(g_{1}, g_{2}, g_{3}\right) \in \mathrm{GL}_{3}(k)$, we have

$$
Q_{\mathcal{J}} \cong-\left[\left(g_{2} g_{3} n_{C}\right) \perp\left(g_{3} g_{1} n_{C}\right) \perp\left(g_{1} g_{2} n_{C}\right)\right] .
$$

Proof. The uniqueness of $Q_{\mathcal{J}}$ follows frome Witt's Theorem. To establish its existence, we may assume that $\mathcal{J}$ is reduced (1.2) and hence has the form $\mathcal{J}=\mathrm{H}_{3}(C, g)$ 
as above. Writing $E$ for the (split) diagonal torus in $\mathcal{J}$, we have $\delta(E / k)=0$, and (1.6.1), (1.6.2), (1.7.1) lead to the desired conclusion.

2.3. Proposition. Let $A$ be an absolutely simple Jordan algebra of degree 3 and dimension 9 over $k$, written as $A=\mathrm{H}(B, *)$ for some central simple associative $k$-algebra $(B, *)$ of degree 3 with involution of the second kind. Then $K=\operatorname{Cent}(B)$ is a subalgebra of Oct $A$. More precisely, we have

$$
n_{\text {Oct } A} \cong n_{K} \perp\left(d_{K / k} Q_{A}\right) .
$$

Proof. It suffices to establish (1) since this implies that $K$ splits Oct $A$ and so must be a subalgebra of it ([4, Lemma 5] and [12, Proposition 4.3]). Let us first assume char $k \neq 2$. Then

$$
\begin{aligned}
<-1>\perp \mathbf{h} \perp Q_{A} & \cong S_{A} \\
& \cong S_{E} \perp\left(\left.S_{A}\right|_{E^{\perp}}\right) \\
& \cong<-d_{E / k}>\perp \mathbf{h} \perp\left(\left.S_{A}\right|_{E^{\perp}}\right)
\end{aligned}
$$

which, by cancelling $\mathbf{h}$ and adding $<1>$, yields

$$
\mathbf{h} \perp Q_{A} \cong n_{k\{\delta(E / k)\}} \perp\left(\left.S_{A}\right|_{E^{\perp}}\right) .
$$

Here we pass to the $\delta(K / k)$-associates and observe (1.1.1), (1.1.2) to conclude

$$
\begin{aligned}
n_{K} \perp\left(d_{K / k} Q_{A}\right) & \cong n_{k\{\delta(E / k)+\delta(K / k)\}} \perp\left(\left.d_{K / k} S_{A}\right|_{E^{\perp}}\right) \\
& \cong n_{\text {Oct } A}
\end{aligned}
$$

We are left with the case char $k=2$. Then

$$
\begin{aligned}
n_{k\{1\}} \perp Q_{A} & \cong S_{A}^{0} \\
& \cong S_{E}^{0} \perp\left(\left.S_{A}\right|_{E^{\perp}}\right) \\
& \cong n_{k\{\delta(E / k)+1\}} \perp\left(\left.S_{A}\right|_{E^{\perp}}\right)
\end{aligned}
$$

This time we pass to the $(\delta(K / k)+1)$-associates and observe $d_{K / k}=1$ to conclude

$$
\begin{aligned}
& n_{K} \perp\left(d_{K / k} Q_{A}\right) \cong n_{K} \perp Q_{A} \\
& \cong n_{k\{\delta(E / k)+\delta(K / k)\}} \perp\left(\left.d_{K / k} S_{A}\right|_{E^{\perp}}\right) \\
& \cong n_{\text {Oct } A} \text {, }
\end{aligned}
$$

as claimed. 
2.4. Clearly, the (hypothetical) reduced model of $A=\mathrm{H}(B, *)$ as in 2.3 must have the form $\mathrm{H}_{3}(K, g)$ for some diagonal matrix $g \in \mathrm{GL}_{3}(k)$. Similary, the coordinate algebra of the (hypothetical) reduced model of an Albert algebra $\mathcal{J}$, given as $\mathcal{J}=$ $\mathcal{J}(B, *, u, \beta)$ by means of the generalized second Tits construction, agrees with the coordinate algebra of $\mathcal{J}$ itself in the sense of $[18,1.9]$, i.e., with Oct $A^{(u)}$ $[18,1.8]$. Keeping this in mind, we can now show that there exists an intimate relationship between the reduced model of an Albert algebra and the one of an arbitrary absolutely simple 9-dimensional subalgebra.

2.5. Proposition. Let $\mathcal{J}$ be an Albert algebra over $k$ and $A$ an absolutely simple 9-dimensional subalgebra of $\mathcal{J}$. Suppose that, for some torus $K / k$ of rank 2 and some $g=\operatorname{diag}\left(g_{1}, g_{2}, 1\right) \in \mathrm{GL}_{3}(k), A_{\text {red }}=\mathrm{H}_{3}(K, g)$ is a reduced model of $A$. Then, writing $C$ for the coordinate algebra of $\mathcal{J}, \mathcal{J}_{\text {red }}=\mathrm{H}_{3}(C, g)$ is a reduced model of $\mathcal{J}$.

Proof. We realize $\mathcal{J}$ as $\mathcal{J}=\mathcal{J}(B, *, u, \beta)$ by means of the generalized second Tits construction in such a way that $A=\mathrm{H}(B, *)$. Then $C \cong$ Oct $A^{(u)}(2.4)$ and $A^{(u)} \cong$ $\mathrm{H}\left(B, *^{(u)}\right)$, where $*^{(u)}$ is the $u$-twist of $*: b \longmapsto u b^{*} u^{-1}$. Hence $K \cong \operatorname{Cent}(B)(2.4)$ is a subalgebra of $C$ (2.3). Setting $\mathcal{J}_{1}=\mathrm{H}_{3}(C, g)$, we must show $\mathcal{J} \otimes_{k} k^{\prime} \cong \mathcal{J}_{1} \otimes_{k} k^{\prime}$ for any reducing field $k^{\prime} / k$ of $\mathcal{J}$. To this end we choose a maximal torus $E \subset A$ and put $E^{\prime}=E \otimes_{k} k^{\prime}$ if this is a field, $E^{\prime}=k^{\prime}$ otherwise. Then $E^{\prime} / k^{\prime}$ is a field extension of degree 1 or 3 , and $E^{\prime} / k$ is a reducing field of $A$ satisfying

$$
\mathcal{J}^{\prime}=\mathcal{J} \otimes_{k} E^{\prime} \supset A \otimes_{k} E^{\prime} \cong A_{\text {red }} \otimes_{k} E^{\prime} \cong \mathrm{H}_{3}\left(K \otimes_{k} E^{\prime}, g\right) .
$$

We now argue as in the proof of [3, Theorem 8] by choosing an isomorphism $\psi: A \otimes_{k} E^{\prime} \stackrel{\sim}{\longrightarrow} \mathrm{H}_{3}\left(K \otimes_{k} E^{\prime}, g\right)$ and using, in standard notation for the hermitian matrix units [6], the quantities

$$
\begin{aligned}
c_{i} & =\psi^{-1}(1[i i]) & (1 \leq i \leq 3), \\
u_{i j} & =\psi^{-1}(1[1 j]) & (j=2,3),
\end{aligned}
$$

to obtain a coordinatization $\mathcal{J}^{\prime} \stackrel{\sim}{\longrightarrow} \mathrm{H}_{3}\left(C \otimes_{k} E^{\prime}, h\right)$ for some $h=\operatorname{diag}\left(h_{1}, h_{2}, 1\right) \in$ $\mathrm{GL}_{3}\left(E^{\prime}\right)$. From this we easily conclude $h=g$ and then

$$
\left(\mathcal{J} \otimes_{k} k^{\prime}\right) \otimes_{k^{\prime}} E^{\prime} \cong\left(\mathcal{J}_{1} \otimes_{k} k^{\prime}\right) \otimes_{k^{\prime}} E^{\prime} .
$$

But since $\mathcal{J} \otimes_{k} k^{\prime}$ and $\mathcal{J}_{1} \otimes_{k} k^{\prime}$ are both reduced, they must be isomorphic by 1.7, 1.2 .

2.6. In our search for reduced models, 2.5 allows us to focus attention on absolutely simple Jordan algebras of degree 3 and dimension 9. In this special case, we can not 
only solve the existence problem but also provide an explicit description. Notice that

$$
d_{K / k} n_{K} \cong-n_{K}
$$

for any torus $K / k$ of rank 2 . In what follows, the symbol "Cay" refers to the (iterated) Cayley-Dickson doubling process.

2.7. Theorem. Let $A$ be an absolutely simple Jordan algebra of degree 3 and dimension 9 over $k$, written as $A=\mathrm{H}(B, *)$ for some central simple associative $k$ algebra $(B, *)$ of degree 3 with involution of the second kind. Then, given arbitrary elements $g_{1}, g_{2} \in k^{\times}$and setting $g=\operatorname{diag}\left(g_{1}, g_{2}, 1\right), K=$ Cent $(B)$, the following statements are equivalent.

(i) $A^{\prime}=\mathrm{H}_{3}(K, g)$ is a reduced model of $A$.

(ii) Oct $A \cong$ Cay $\left(K ;-g_{1},-g_{2}\right)$.

Proof. We put $C=$ Cay $\left(K ;-g_{1},-g_{2}\right)$ and compute

$$
\begin{aligned}
n_{C} & \cong n_{K} \perp\left(g_{1} n_{K}\right) \perp\left(g_{2} n_{K}\right) \perp\left(g_{1} g_{2} n_{K}\right) \\
& \cong n_{K} \perp d_{K / k}\left[-\left(\left(g_{1} n_{K}\right) \perp\left(g_{2} n_{K}\right) \perp\left(g_{1} g_{2} n_{K}\right)\right)\right] \\
& \cong n_{K} \perp d_{K / k} Q_{A^{\prime}}
\end{aligned}
$$

Suppose first that (i) holds. Then $Q_{A} \cong Q_{A^{\prime}}(1.2)$, which implies

$$
n_{C} \cong n_{K} \perp d_{K / k} Q_{A} \cong n_{\text {Oct } A}
$$

and then $C \cong$ Oct $A$. Conversely, if (ii) holds, Witt cancellation and 2.3 yield $Q_{A} \cong Q_{A^{\prime}}$. Hence 1.7 gives $A \otimes_{k} k^{\prime} \cong A^{\prime} \otimes_{k} k^{\prime}$ for any reducing field $k^{\prime} / k$ of $A$, as desired.

Combining 2.7 with $2.3,2.5$, we obtain the following final result.

2.8. Theorem. Let $\mathcal{J}$ be an absolutely simple Jordan algebra of degree 3 over $k$. Then $\mathcal{J}$ has a reduced model, uniquely determined up to isomorphism and written as $\mathcal{J}_{\text {red }}$.

2.9. The reduced model of an Albert algebra $\mathcal{J}$ over $k$ looks like this. Realize $\mathcal{J}$ by means of the generalized second Tits construction as $\mathcal{J}=\mathcal{J}(B, *, u, \beta)$ and 
put $A=\mathrm{H}(B, *)$. Then $K=$ Cent $(B)$ is a subalgebra of Oct $A^{(u)}$ (2.3), so some $g_{1}, g_{2} \in k^{\times}$have Oct $A^{(u)} \cong$ Cay $\left(K ; g_{1}, g_{2}\right)$. Now $2.4,2.5,2.7$ yield

$$
\mathcal{J}_{\text {red }} \cong \mathrm{H}_{3}\left(\text { Oct } A^{(u)}, g\right), \quad g=\operatorname{diag}\left(-g_{1},-g_{2}, 1\right) .
$$

2.10. We close this section by two applications. First, assume char $k \neq 2$ and let $\mathcal{J}$ be an Albert algebra over $k$. We define the invariants $\bmod 2$ of $\mathcal{J}$ to be the invariants mod 2 of $\mathcal{J}_{\text {red }}$ in the sense of Serre [25], Rost [22] or Petersson-Racine [19]. This definition is equivalent to the one given by Serre [25] and Rost [22] since, for any reducing field $k^{\prime} / k$ of $\mathcal{J}$ whose degree is odd, the restriction map

$$
H^{i}(k, \mathbf{Z} / 2 \mathbf{Z}) \longrightarrow H^{i}\left(k^{\prime}, \mathbf{Z} / 2 \mathbf{Z}\right) \quad(i \geq 0)
$$

as injective.

Next, we characterize absolutely simple Jordan algebras of degree 3 and dimension 9 whose octonion algebra is split.

2.11. Theorem. Let $A$ be an absolutely simple Jordan algebra of degree 3 and dimension 9 over $k$. Then the following statements are equivalent.

(i) $A_{\text {red }}$ contains nilpotent elements other than zero.

(ii) Oct $A$ splits.

(iii) $S_{A}$ has maximal Witt index.

(iv) If $A \cong \mathrm{H}(B, *)$ for some central simple associative $k$-algebra $(B, *)$ of degree 3 with involution of the second kind and $K=$ Cent $(B)$, then

$$
S_{A} \cong<-d_{K / k}>\perp \mathbf{h}^{4}
$$

Moreover, all these conditions are fulfilled if $A$ is a first Tits construction.

Proof. (i) $\Longleftrightarrow$ (ii). $A_{\text {red }}$ contains nilpotent elements other than zero iff it is isomorphic to $\mathrm{H}_{3}\left(K, g_{\text {nil }}\right), g_{\text {nil }}=\operatorname{diag}(1,-1,1)(1.7)$ iff Oct $A$ is isomorphic to Cay $(K ;-1,1)(2.7)$ iff Oct $A$ splits.

(ii) $\Longrightarrow$ (iv). We first consider the case char $k \neq 2$. Then (1.11.2) implies

$$
d_{K / k} S_{A} \cong<-1>\perp n_{\text {Oct } A} \cong<-1>\perp \mathbf{h}^{4},
$$

forcing $S_{A} \cong<-d_{K / k}>\perp \mathbf{h}^{4}$, and we are left with the case char $k=2$. Since Oct $A$ splits, we conclude, abbreviating $n=n_{\text {Oct } A}$ :

$$
S_{A}^{0} \cong n_{\delta(K / k)+1}
$$




$$
\begin{aligned}
& \cong\left(\mathbf{h}^{4}\right)_{\delta(K / k)+1} \cong\left(n_{k\{0\}} \perp \mathbf{h}^{3}\right)_{\delta(K / k)+1} \\
& \cong n_{k\{\delta(K / k)+1\}} \perp \mathbf{h}^{3}
\end{aligned}
$$

Setting $E=k \oplus K$, this implies

$$
\begin{aligned}
& S_{A} \cong<1>\perp S_{A}^{0} \\
& \cong<1>\perp n_{k\{\delta(K / k)+1\}} \perp \mathbf{h}^{3} \\
& \cong<1>\perp S_{E}^{0} \perp \mathbf{h}^{3} \\
& \cong S_{E} \perp \mathbf{h}^{3} \\
& \cong<-d_{K / k}>\perp \mathbf{h}^{4}
\end{aligned}
$$

(iv) $\Longrightarrow$ (iii). Obvious.

(iii) $\Longrightarrow$ (ii). $S_{A}$ having maximal Witt index implies that $\left.S_{A}\right|_{E^{\perp}}$, for any maximal torus $E \subset A$, is isotropic. This property carries over to $n_{\text {Oct } A}$ by 1.11 .1 , so Oct $A$ splits.

It remains to show that all four conditions are fulfilled if $A$ is a first Tits construction (1.9), i. e., $A=\mathcal{J}(E, \alpha)$ for some torus $E / k$ of rank 3 and $\alpha \in k^{\times}$. But then, by 1.6.1 and 1.9.1, $S_{A}$ has Witt index 1,3 on $E, E^{\perp}$, respectively, and (iii) follows.

2.12. If $A$ as in 2.11 (iv) is a reduced first Tits construction, we combine 2.11 with 1.7 to conclude $A \cong \mathrm{H}_{3}(K$, $\operatorname{diag}(1,-1,1))$ and thus recover [14, Theorem 3]. Finally, one may ask whether the property of an absolutely simple Jordan algebra

of degree 3 and dimension 9 to be a first Tits construction is actually equivalent to conditions (i) - (iv) in 2.11. We have been unable to settle this question.

\section{Octonions and Jordan algebras of degree 3}

3.1. In this section we fix an absolutely simple Jordan algebra $A$ of degree 3 and dimension 9 over $k$, written as $A=\mathrm{H}(B, *)$ for some central simple associative $k$-algebra $(B, *)$ of degree 3 with involution of the second kind. As before, we put $K=\operatorname{Cent}(B)$.

Our aim is to characterize internally the octonion algebra of $A$ in terms of $A$ itself. 
This has been done previously for the octonion norm (cf. 1.11) but doing it for the octonion algebra is a different matter.

3.2. We begin by recalling some facts from the theory of quadratic forms that are well known over fields of characteristic not 2 but, when properly phrased, retain their validity over an arbitrary $\operatorname{ring} R$. Let $M, N$ be $R$-modules and $q: M \longrightarrow R$ (resp. $b: N \times N \longrightarrow R$ ) a quadratic (resp. symmetric bilinear) form over $R$. Then there exists a unique quadratic form

$$
q \otimes b: M \otimes N \longrightarrow R
$$

satisfying

$$
\begin{aligned}
(q \otimes b)(v \otimes w) & =q(v) b(w, w), \\
(q \otimes b)\left(v \otimes w, v^{\prime} \otimes w^{\prime}\right) & =q\left(v, v^{\prime}\right) b\left(w, w^{\prime}\right)
\end{aligned}
$$

for all $v, v^{\prime} \in M, w, w^{\prime} \in N[23]$.

3.3. Example. For a symmetric $n \times n$-matrix $S$ over $R(n \in \mathbf{N})$ we denote by $<S>_{\text {bil }}$ the symmetric bilinear form on $R^{n}$ determined by

$$
<S>_{\text {bil }}(x, y)={ }^{t} x S y
$$

for $x, y \in R^{n}$. Given $a_{1}, \ldots, a_{n} \in R$ and abbreviating

$$
<a_{1}, \ldots, a_{n}>_{\text {bil }}=<\operatorname{diag}\left(a_{1}, \ldots, a_{n}\right)>_{\text {bil }} \text {, }
$$

one obtains, for any quadratic form $q: M \longrightarrow R$ over $R$, an isometry

$$
q \otimes<a_{1}, \ldots, a_{n}>_{\text {bil }} \cong\left(a_{1} q\right) \perp \ldots \perp\left(a_{n} q\right)
$$

via the canonical identification $M \otimes R^{n} \cong M^{n}$.

3.4. Let $R$ be a ring, $R^{\prime}$ a (unital commutative associative) $R$-algebra and $\lambda$ : $R^{\prime} \longrightarrow R$ an $R$-linear form. For a quadratic (resp. symmetric bilinear) form $q^{\prime}$ : $M^{\prime} \longrightarrow R^{\prime}$ (resp. $b^{\prime}: N^{\prime} \times N^{\prime} \longrightarrow R^{\prime}$ ) over $R^{\prime}$ we denote by $\lambda_{*} q^{\prime}=\lambda \circ q^{\prime}: M^{\prime} \longrightarrow R$ (resp. $\lambda_{*} b^{\prime}=\lambda \circ b^{\prime}: N^{\prime} \times N^{\prime} \longrightarrow R$ ) the Scharlau transfer of $q^{\prime}$ (resp. $b^{\prime}$ ) relative to $\lambda[24$, p. 47], which is a quadratic (resp. symmetric bilinear) form over $R$. Just as in [24, Chap. 2, 5.6] one checks the following result.

3.5. Theorem. (Scharlau). Let $R$ be a ring, $R^{\prime}$ an $R$-algebra and $\lambda: R^{\prime} \longrightarrow R$ an $R$-linear form. Then, given a quadratic form $q: M \longrightarrow R$ over $R$ and a symmetric bilinear form $b^{\prime}: N^{\prime} \times N^{\prime} \longrightarrow R^{\prime}$ over $R^{\prime}$, there exists a canonical isometry

$$
\lambda_{*}\left(\left(q \otimes_{R} R^{\prime}\right) \otimes_{R^{\prime}} b^{\prime}\right) \cong q \otimes_{R}\left(\lambda_{*} b^{\prime}\right) .
$$


In particular, we have

$$
\lambda_{*}\left(q \otimes_{R} R^{\prime}\right) \cong q \otimes_{R}\left(\lambda_{*}<1>_{\mathrm{bil}}\right)
$$

3.6. We now return to the situation described in 3.1 and pick any maximal torus $E \subset A$. Excluding finite base fields of certain low cardinalities, Lemmata $3.4-3.9$ in [16] show that $A$ may be obtained from $E$ by means of the toral Tits process: There exist a torus $L / k$ of rank 2 (with canonical involution ${ }^{-}$) as well as invertible elements $v \in E, \gamma \in L$ satisfying $N_{E}(v)=\gamma \bar{\gamma}$ and $A \cong J(E, v, \gamma, L)$ (cf. 1.10). Conversely, if $A$ is realized by means of the toral Tits process as above, which can always be achieved by working with an appropriate $E[16,3.1]$, we are able to express its octonion algebra in terms of the parameters involved. To see this, we recall that, over a field of characteristic 2 , every nondegenerate symmetric bilinear form is either alternating or diagonalizable [1], the former possibility of course being excluded if the dimension is odd.

3.7. Theorem. Let $L / k, E / k$ be tori of rank 2,3, respectively, and $v \in E, \gamma \in L$ be invertible elements satisfying $N_{E}(v)=\gamma \bar{\gamma}$, where ${ }^{-}$stands for the canonical involution of $L$. Setting $A=\mathcal{J}(E, v, \gamma, L)$ in the sense of the toral Tits process (1.10) and choosing any diagonalization

$$
T^{(v)} \cong<h_{1}, h_{2}, h_{3}>_{\text {bil }} \quad\left(h_{i} \in k^{\times}\right)
$$

of the symmetric bilinear form $T^{(v)}$ on E given by

$$
T^{(v)}\left(w, w^{\prime}\right)=T_{E}\left(v w w^{\prime}\right) \quad\left(w, w^{\prime} \in E\right),
$$

the octonion algebra of $A$ may then be derived from $L$ via

$$
\text { Oct } A \cong \operatorname{Cay}\left(L ;-d_{E / k} h_{1},-d_{E / k} h_{2}\right)
$$

as a twofold application of the Cayley-Dickson doubling process.

Proof. Setting $n=n_{\text {Oct } A},(1.10 .1)$ and (1.11.1) yield

$$
n \cong n_{L} \perp\left(\left.d_{K / k} S_{A}\right|_{E^{\perp}}\right)
$$

Next observe

$$
\operatorname{det} T^{(v)}=N_{E}(v)\left(\operatorname{det} T_{E}\right)=d_{E / k} n_{L}(\gamma)
$$

whence

$$
h_{3} \equiv d_{E / k} h_{1} h_{2} n_{L}(\gamma) \bmod k^{\times 2} .
$$


Finally, by the precise nature of the toral Tits process, $E^{\perp}$ identifies with $L \otimes_{k} E$ under $(0, b)=b$ for $b \in L \otimes_{k} E$, and (1.8.1) shows

$$
\left.S_{A}\right|_{E^{\perp}}=\lambda_{*} n_{L \otimes_{k} E},
$$

where $\lambda$ is the linear form on $E$ given by

$$
\lambda(w)=-T_{E}(v w) \quad(w \in E) .
$$

Summing up, we therefore have

$$
\begin{aligned}
n \cong & n_{L} \perp\left(d_{K / k} \lambda_{*}\left(n_{L} \otimes_{k} E\right)\right) \\
\cong & \left.n_{L} \perp\left(d_{K / k} n_{L} \otimes_{k} \lambda_{*}<1>_{\text {bil }}\right)\right) \\
\cong & n_{L} \perp\left(-d_{K / k} n_{L} \otimes_{k} T^{(v)}\right) \\
\cong & n_{L} \perp\left(-d_{K / k} h_{1} n_{L}\right) \perp\left(-d_{K / k} h_{2} n_{L}\right) \perp \\
& \left(-d_{K / k} d_{E / k} h_{1} h_{2} n_{L}\right) \\
\cong & n_{L} \perp\left(d_{K / k} d_{L / k} h_{1} n_{L}\right) \perp\left(d_{K / k} d_{L / k} h_{2} n_{L}\right) \perp \\
& \left(d_{K / k} d_{E / k} d_{L / k} h_{1} h_{2} n_{L}\right) \\
\cong & n_{L} \perp\left(d_{E / k} h_{1} n_{L}\right) \perp\left(d_{E / k} h_{2} n_{L}\right) \perp\left(d_{E / k}^{2} h_{1} h_{2} n_{L}\right) \\
\cong & n_{C},
\end{aligned}
$$

where $C=$ Cay $\left(L ;-d_{E / k} h_{1},-d_{E / k} h_{2}\right)$. Since an octonion algebra is uniquely determined by its norm, the assertion follows.

3.8. The Cayley-Dickson doubling process is not the only way to construct octonions. Another approach, recently employed by Knus-Parimala-Sridharan [7] and already implicit in [5], works with ternary hermitian spaces. We sketch the details. Let $K / k$ be a torus of rank 2 and $(V, h)$ a ternary $K / k$-hermitian space, so $V$ is a projective $K$-module of rank 3 and $h: V \times V \longrightarrow K$ is a nondegenerate hermitian form, linear in the first variable, antilinear in the second. We assume that $(V, h)$ has trivial determinant, i.e., $\wedge^{3}(V, h) \cong(K,<1>)$ where $<1>$ stands for the hermitian form $a \bar{b}$ on $K$. Choosing an isomorphism $\alpha: \wedge^{3}(V, h) \stackrel{\sim}{\longrightarrow}(K,<1>)$, we obtain an induced hermitian vector product on $V$ associating with any two $v, w \in V$ the unique element $v \times w \in V$ determined by

$$
h(u, v \times w)=\alpha(u \wedge v \wedge w) \quad(u \in V) .
$$

Then the vector space $K \oplus V$ over $k$ under the multiplication

$$
(a \oplus v)(b \oplus w)=(a b-h(v, w)) \oplus(a w+\bar{b} v+v \times w)
$$


for $a, b \in K, v, w \in V$ becomes an octonion algebra $C$ over $k$ whose norm is given by

$$
n_{C}((a, v))=n_{K}(a)+h(v, v) .
$$

Notice that $n_{C}$ does not depend on $\alpha$. Hence, up to isomorphism, neither does $C$, and we are allowed to write $C=$ Cay $(K ; V, h)$.

3.9. Theorem. Let $A=\mathcal{J}(E, v, \gamma, L)$ be a toral Tits process as in 3.7. Viewing $V=E \otimes_{k} L$ as an $L$-module, setting $T=T_{E \otimes_{k} L / L}$ and defining $h: V \times V \longrightarrow L$ by

$$
h\left(w, w^{\prime}\right)=-d_{E / k} d_{L / k} T\left(w v w^{\prime *}\right) \quad\left(w, w^{\prime} \in V\right),
$$

$(V, h)$ is a ternary hermitian $L / k$-space with trivial determinant, and we have

$$
\text { Oct } A \cong \operatorname{Cay}(L ; V, h) \text {. }
$$

Proof. We choose a basis of $E / k$ and compute mod. $n_{L}\left(L^{\times}\right)$to obtain

$$
\begin{aligned}
\operatorname{det} h & \equiv-d_{E / k} d_{L / k} N_{E}(v) d_{E / k} \\
& \equiv-d_{L / k} n_{L}(\gamma) \\
& \equiv 1 \bmod n_{L}\left(L^{\times}\right)
\end{aligned}
$$

Thus $C=$ Cay $(L ; V, h)$ exists (3.8). Writing $Q$ for the $k$-quadratic form $w \longmapsto$ $h(w, w)$ on $V$ and identifiying $E^{\perp}=V$ canonically in $A$, we apply (1.10.1), (1.10.2) and (1.11.1) to conclude

$$
\begin{aligned}
n_{\text {Oct } A} & \cong n_{L} \perp\left(\left.d_{E / k} d_{L / K} S_{A}\right|_{V}\right) \\
& \cong n_{L} \perp Q \\
& \cong n_{C}
\end{aligned}
$$

as desired. 


\section{Examples: The unital Tits process}

4.1. Returning to 1.8 , we let $K / k$ be a torus of rank $2, B$ a separable associative $K$-algebra of degree 3 , with norm $N$, trace $T$ and adjoint $\#, *$ a $K / k$-involution of $B$ and $u \in A=\mathrm{H}(B, *), \beta \in K$ invertible elements satisfying $N(u)=\beta \beta^{*}$.

In the special case $u=1$ the relation $\beta \beta^{*}=1$ amounts to $\beta=\theta / \theta^{*}$ for some $\theta \in K^{\times}$. We then refer to

$$
\mathcal{J}\left(K, B, *, 1, \theta / \theta^{*}\right)
$$

as the unital Tits process. The unital Tits process is not too far removed from the ordinary Tits process. Indeed, $[15 ; 3.7,3.9]$ (see also $[18,1.5]$ ) are easily seen to imply

$$
\mathcal{J}(K, B, *, u, \beta)^{(u)} \cong \mathcal{J}\left(K, B, *^{(u)}, 1, \theta / \theta^{*}\right),
$$

where $\theta=\beta^{*}$ and $*^{(u)}$ stands for the $u$-twist $b \longmapsto u b^{*} u^{-1}$ of $*$. In particular, we have

4.2. Propositon. Up to isotopy, all Albert algebras are obtained by the unital Tits process.

4.3. We wish to construct Albert division algebras by means of the unital Tits process. This will be accomplished in two steps, the first one yielding examples of absolutely simple 9-dimensional Jordan division algebras of degree 3 by the same method. We begin by deriving an expanded version of 3.7 for the unital Tits process.

4.4. Lemma. Let $E / k$ be a torus of rank 3. Then

$$
T_{E} \cong\left\{\begin{array}{l}
<1,2,2 d_{E / k}>_{\text {bil }} \text { for } \text { char } k \neq 2, \\
<1,1,1>_{\text {bil }} \text { for char } k=2 .
\end{array}\right.
$$

Proof. For char $k \neq 2$, we need only apply Springer's Extension Theorem (1.2) to reduce to the case that $E$ contains zero divisors, and then the assertion is straightforward to check. For char $k=2$, we set $T=T_{E}, S=S_{E}$, have the orthogonal splitting $E=k 1 \perp E^{0}$ relative to $T$ and conclude from (1.5.1) that $T^{0}=S^{0}$ (the right-hand side viewed as a symmetric bilinear form) is alternating 
on $E^{0} \times E^{0}$. Hence we can find a hyperbolic pair $(u, v)$ of $\left(E^{0}, T^{0}\right)$, and one checks easily that the basis

$$
e_{1}=1+u, e_{2}=1+v, e_{3}=1+u+v
$$

of $E / k$ satisfies $T\left(e_{i}, e_{j}\right)=\delta_{i j}(1 \leq i, j \leq 3)$, as claimed.

4.5. Theorem. Let $L / k, E / k$ be tori of rank 2,3 , respectively, and $\theta \in L^{\times}$. Write - for the canonical involution of $L / k$ and put

$$
K=k\{\delta(L / k)+\delta(E / k)\}
$$

Then the octonion algebra and the reduced model of the unital toral Tits process

$$
A=\mathcal{J}(E, 1, \theta / \bar{\theta}, L)
$$

may be described as follows. For char $k \neq 2$, we have

Oct $A \cong \operatorname{Cay}\left(L ;-2,-d_{E / k}\right), \cong \operatorname{Cay}\left(K ;-2,-d_{E / k}\right), A_{\text {red }} \cong \mathrm{H}_{3}\left(K, \operatorname{diag}\left(2, d_{E / k}, 1\right)\right)$

whereas, for char $k=2$, Oct $A$ splits and

$$
A_{\text {red }} \cong \mathrm{H}_{3}(K, \operatorname{diag}(1,-1,1)) \text {. }
$$

Proof. We first treat the case char $k \neq 2$. Then 3.7 for $v=1$ combined with 4.4 yields

$$
\text { Oct } A \cong \operatorname{Cay}\left(L ;-d_{E / k},-2 d_{E / k}\right) \cong \operatorname{Cay}\left(L ;-2,-d_{E / k}\right) .
$$

Using (1.10.2) we conclude that

$$
n_{\text {Oct } A} \cong<1,-d_{L / k}, 2,-2 d_{L / k}, d_{E / k},-d_{K / k}, 2 d_{E / k},-2 d_{K / k}>
$$

up to isometry is symmetric in $K$ and $L$. Hence Oct $A \cong \operatorname{Cay}\left(K ;-2,-d_{E / k}\right)$, and the formula for the reduced model drops out of $2.7,2.4$ and (1.10.1). We are left with the case char $k=2$. Arguing as before, we obtain Oct $A \cong \operatorname{Cay}(L ; 1,1)$, which is obviously split. The rest now follows from 2.11. and 1.7.

4.6. Example. Put $k=\mathbf{R}, L=\mathbf{C}, E=\mathbf{R} \oplus \mathbf{R} \oplus \mathbf{R}$. Then $A$ as in 4.5 is reduced, Oct $A \cong \mathbf{O}$ is the real octonion division algebra and $A \cong A_{\text {red }} \cong \mathrm{H}_{3}(\mathbf{C})$.

4.7. Returning to 4.3 , we consider an arbitrary base field $F$, char $F \neq 2$, and denote by - the $F$-involution given on the polynomial ring $F[s, t]$ as well as on the rational function field $L=F(s, t)$ by

$$
\bar{s}=t, \bar{t}=s .
$$


Setting

$$
k=\mathrm{H}\left(L,{ }^{-}\right)
$$

the elementary symmetric funtions

$$
s_{1}=s+t, t_{1}=s t
$$

are algebraically independent over $F$ and satisfy

$$
F[s, t] \cap k=F\left[s_{1}, t_{1}\right], k=F\left(s_{1}, t_{1}\right) .
$$

Furthermore, $L / k$ is a separable quadratic extension, with non trivial Galois automorphism - ${ }^{-}$, whose discriminant is easily seen to be given by

$$
d_{L / k} \equiv\left(s_{1}^{2}-4 t_{1}\right) \bmod k^{\times 2} \equiv(s-t)^{2} \bmod k^{\times 2} .
$$

4.8. Theorem. In the situation of 4.7 , let $E_{0} / F$ be a separable cubic field extension and put $E=E_{0} \otimes_{F} k$. Then the unital toral Tits process

$$
A=\mathcal{J}(E, 1, s / t, L)
$$

is a division algebra over $k$. Writing $(B, *)$ for the central simple associative $k$ algebra of degree 3 with involution of the second kind satisfying $A \cong \mathrm{H}(B, *), K=$ $\operatorname{Cent}(B)$ is a field, and, putting

$$
C_{0}=\operatorname{Cay}\left(F ;-2,-d_{E_{0} / F}\right),
$$

we have

$$
\text { Oct } A \cong \text { Cay }\left(C_{0} \otimes_{F} k, s_{1}^{2}-4 t_{1}\right) \text {. }
$$

Moreover, Oct $A$ splits over $k$ if and only if $C_{0}$ splits over $F$.

Proof. We have $E \otimes_{k} L \cong E_{0} \otimes_{F} F(s, t)$, and one checks easily that $s / t$ is not a generic norm of $E \otimes_{k} L / L$. Hence $A$ is a division algebra. Furthermore,

$$
\begin{aligned}
d_{K / k} & \equiv d_{E / k} d_{L / k} \bmod k^{\times 2} \\
& \equiv d_{E_{0} / F}\left(s_{1}^{2}-4 t_{1}\right) \bmod k^{\times 2}
\end{aligned}
$$

and an elementary discussion shows that this cannot be a square in $k$. Thus $K$ is a field. Setting $d_{0}=d_{E_{0} / F}, 4.4$ implies

$$
\begin{aligned}
n_{\text {Oct } A} & \cong n_{L} \perp 2 n_{L} \perp d_{0} n_{L} \perp 2 d_{0} n_{L} \\
& \cong<1,2, d_{0}, 2 d_{0}>\perp\left[-\left(s_{1}^{2}-4 t_{1}\right)<1,2, d_{0}, 2 d_{0}>\right] \\
& \cong n_{C^{\prime}}, C^{\prime}=\operatorname{Cay}\left(C_{0} \otimes_{F} k, s_{1}^{2}-4 t_{1}\right),
\end{aligned}
$$


hence Oct $A \cong C^{\prime}$. Finally, if $C_{0}$ splits so obviously does Oct $A$. Conversely, suppose Oct $A$ splits but $C_{0}$ doesn't. Then $n_{0}$, the norm of $C_{0}$, is anisotropic and represents $s_{1}^{2}-4 t_{1}$ over $k=F\left(t_{1}\right)\left(s_{1}\right)$, hence over $F\left(t_{1}\right)\left[s_{1}\right]$ [24, Chap. 4, 3.2]. Relative to some $F$-basis $\left(e_{i}\right)$ of $C_{0}$ we can therefore find a family $\left(\varphi_{i}\right)$ of elements in $F\left(t_{1}\right)\left[s_{1}\right]$ satisfying

$$
n_{0}\left(\sum e_{i} \otimes \varphi_{i}\right)=s_{1}^{2}-4 t_{1} .
$$

Here the specialization $s_{1} \longrightarrow 0, t_{1} \longrightarrow t_{1} / 4 s_{1}$ is allowed and shows that $n_{0}$ represents $s_{1} / t_{1}$ over $k$, which just as before is seen to be a contradiction. This completes the proof.

4.9. We now start from a central simple associative algebra of degree 3 with involution of the second kind as in 4.8, pass to a rational function field over $k$ and perform the unital Tits process again to arrive at the following result.

4.10. Theorem. Let $(B, *)$ be a central associative division algebra of degree 3 over $k$ with involution of the second kind and $K=$ Cent $(B)$. Then, for every $\theta \in K-k$, the unital second Tits construction

$$
\mathcal{J}=\mathcal{J}\left(B \otimes_{K} K(X), *, 1, \frac{X-\theta}{X-\theta^{*}}\right)
$$

is an Albert division algebra over the rational function field $k(X)$. Moreover, setting $A=\mathrm{H}(B, *)$, the coordinate algebra of $\mathcal{J}$ is

$$
(\text { Oct } A) \otimes_{k} k(X) \text {. }
$$

Proof. One checks easily that the generic norm of $B$ does not represent $(X-$ $\theta) /\left(X-\theta^{*}\right)$ over $K(X)$. Hence $\mathcal{J}$ is a division algebra. By $[18,1.8]$ it has the coordinate algebra $($ Oct $A) \otimes_{k} k(X)$.

4.11. Example. Let $F$ be a field of characteristic not 2 and $E_{0} / F$ a seperable cubic field extension such that the quaternion algebra

$$
C_{0}:=\operatorname{Cay}\left(F ;-2,-d_{E_{0} / F}\right)
$$

doesn't split. (For instance, suppose $E_{0}$ is a cubic Galois extension of $F=\mathbf{Q}$; this implies $C_{0} \otimes_{\mathbf{Q}} \mathbf{R} \cong \mathbf{H}$ so, indeed, $C_{0}$ doesn't split.) Then 4.8 yields a central associative division algebra $(B, *)$ of degree 3 with involution of the second kind over $k=F\left(s_{1}, t_{1}\right)$ (cf. 4.7) such that, setting $A=\mathrm{H}(B, *)$, the octonion algebra of $A$ doesn't split. Applying 4.10, we thus obtain an Albert division algebra $\mathcal{J}$ over the rational function field $k(X)$ whose octonion algebra is isomorphic to (Oct $A$ ) $\otimes_{k}$ $k(X)$ and hence doesn't split, either. In particular, $\mathcal{J}$ must be a pure second Tits 
construction. Summing up, we have arrived at an example of a pure second Tits construction Albert division algebra which is arguably more accessible than the one given by Albert [2] as well as the Albert algebra of generic matrices [11].

Note added in proof. In the mean time, Haile, Knus, Rost, and Tignol have answered question 2.12 affirmatively. For this and related results, see their joint paper Algebras of odd degree with involution, trace forms and dihedral extensions (Theorem 16), to appear in Israel J. Math.

\section{References}

1. Albert, A. A.: Symmetric and alternate matrices over an arbitrary field. I. Trans. Amer. Math. Soc. 43, 386 - 436 (1938).

2. $\quad$ - $\quad$ On exceptional Jordan division algebras. Pacific J. Math. 15, $377-404$ (1965).

3. Albert, A. A., Jacobson, N.: On reduced exceptional simple Jordan algebras. Ann. of Math. (2) 66, 400 - 417 (1957).

4. $\quad$ Ferrar, J. C.: Generic splitting fields for composition algebras. Trans. Amer. Math. Soc. 128, 506 - 514 (1967).

5. Jacobson, N.: Composition algebras and their automorphism. Rend. Cir. Mat. di Palermo (II), 7, 55 - 80 (1958).

6. - $\quad$ - $\quad$ "Structure and representations of Jordan algebras.". Amer. Math. Coll. Publ. 39, Providence R I, 1968.

7. Knus, M. A., Parimala, R., Sridharan, R.: On compositions and triality. J. reine angew. Math. 457, $45-70$ (1994).

8. Loos, O.: $\quad$ Separable quadratic algebras acting on pointed quadratic forms. To appear.

9. McCrimmon, K.:The Freudenthal-Springer-Tits constructions of exceptional Jordan algebras. Trans. Amer. Math. Soc. 139, $495-510$ (1969).

10. Petersson, H. P.: Reduced simple Jordan algebras of degree 3 over a field with a discrete valuation. Archiv Math. 25, 593 - 597 (1974). 
11. - $\quad$ Exceptional simple Jordan algebras of generic matrices. Preprint.

12. Petersson, H. P., Racine, M. L.: Springer forms and the first Tits construction of exceptional Jordan division algebras. Manuscripta math. 45, 249 - 272 (1984).

13. $\quad-\quad$ Cubic subfields of exceptional simple Jordan algebras. Proc. Amer. Math. Soc. 91 (1), 31 - 36 (1984).

14. - $\quad$ The toral Tits process of Jordan algebras. Abh. Math. Sem. Univ. Hamburg 54, 251 - 256 (1984).

15. $\quad$ - Jordan Algebras of degree 3 and the Tits process.. J. Algebra 98 (1), 211 - 243 (1986).

16. $\quad$ - Classification of algebras arising from the Tits process. J. Algebra 98, $244-279$ (1986).

17. $\quad-\quad$ Pure and generic first Tits constructions of exceptional Jordan division algebras. Alg. Grps. Geom. 3, 386 - 398 (1986).

18. $\quad-\quad$ On the invariants mod 2 of Albert algebras. J. Algebra 174 (1995), 1049 - 1072.

19. $\quad$ - $\quad$ Albert algebras. In: W. Kaup, et al. (eds.): Jordan algebras, Oberwolfach, 1992. Berlin New York: de Gruyter 1994.

20. - $\quad$ Enumeration and classification of Albert algebras: Reduced models and the invariants mod 2. Proc. Conf. Nonassoviative Algebras, Oviedo, 1993. To appear.

21. Racine, M. L.: A note on quadratic Jordan algebras of degree 3. Trans. Amer. Math. Soc. 164, 93 - 103 (1972).

22. Rost, M.: $\quad$ A descent property of Pfister forms. Preprint, 1991.

23. Sah, C. H.: $\quad$ Symmetric bilinear forms and quadratic forms. J. Algebra 20, $144-160$ (1972). 
24. Scharlau, W.: "Quadratic and hermitian forms". (Grundlehren Math. Wiss. Bd. 270) Berlin Heidelberg New York Tokyo: Springer 1985.

25. Serre, J. - P.: Résumé des cours de l'année 1990 - 91. Annuaire du Collège de France. 